BULLETIN OF THE

AMERICAN MATHEMATICAL SOCIETY

Volume 80, Number 4, July 1974

\title{
CHARACTERS OF CONNECTED LIE GROUPS ${ }^{1}$
}

\author{
BY L. PUKANSZKY
}

Communicated by Jacob Feldman, October 12, 1973

Introduction. If $G$ is a finite group, any irreducible unitary representation of $G$ gives rise to a homomorphism of the group algebra $A$ (=formal linear combinations of the group elements with complex coefficients) the kernel of which is a 2-sided prime ideal, onto the full matrix algebra of the same dimension, and conversely. In this fashion, there is a canonical bijection between the set of all 2-sided prime ideals of $A$ and characters of $G$. Let now $G$ be a separable locally compact group. As generalization to this case of the group algebra we take the group $C^{*}$ algebra $C^{*}(G)$ (cf. $[3,13.9$, p. 270]), and as characters, following the definition, inspired by the pioneering work of R. Godement [6], [7], of A. Guichardet, the characters of $C^{*}(G)$ (cf. [8] or [3, 6.7, p. 126], and 1 below). Then every closed 2-sided prime ideal is primitive, or is the kernel of a factor representation, and conversely (cf. [2], Corollaires 1 and 3, p. 100]). Denoting by $\operatorname{Prim}(G)$ the set of all primitive ideals of $C^{*}(G)$, the question one is led to ask is whether the correspondence character $\mapsto$ primitive ideal establishes a bijection between the set of characters and $\operatorname{Prim}(G)$. By virtue of results of J. Glimm (cf. [5] or [3, 9.1, Théorème, (i) $\Rightarrow$ (iv), p. 169]), in particular, the answer is yes for any type $I$ group $G$. On the other hand Guichardet showed that countable infinite groups, in general, fail badly to have the said property [8, Proposition 2, p. 62].

The principal result of this note (cf. Theorem 1 below) states that the answer is again positive for any connected Lie group $G$. As a corollary, it solves the problem of the existence of characters, established until now only in an incomplete fashion for some special cases, as that of unimodular or solvable groups (cf. [3, 18.7.9, p. 326] and [9, Corollary 7.2, p. 594]). Our proof implies also that if $G$ is solvable and simply connected, the factor representations constructed in [9, Theorem 2, p. 551]) by extending the geometrical approach, applied in the type I case by L. Auslander and B. Kostant [1] indeed provide the set of characters of $G$, as conjectured by the author (cf., e.g., [9, p. 463]). We believe that the essence of the

AMS (MOS) subject classifications (1970). Primary 22D25, 22E45; Secondary 46L05, 46L25.

1 This work has been supported by National Science Foundation grant no. 28976X2. 
results presented here can be best interpreted as a corroboration under some regularity assumption of the idea, due to R. Godement, that for continuous groups in general, the role of irreducible representations as "elementary objects" of representation theory is taken over by the factor representations with trace.

The proofs are long and make much use of previous papers by the author (cf. [9], and parts of [10] and [11] respectively).

2. Let $A$ be a separable $C^{*}$ algebra. We shall say that a factor representation $T$ of $A$ is normal if the factor $R(T)$ it generates is semifinite and, $\Phi$ denoting a faithful normal trace on $R(T)$, the function $a \mapsto \Phi(T(a))$ on $A^{+}$(=character of $T$ ) assumes nonzero finite values. This property is clearly invariant under quasi-equivalence; we denote by $\tilde{A}_{\text {norm }}$ the corresponding subset of the quasi-dual $\tilde{A}$ (for the latter cf. [3, 7.2, p. 136]). The kernel, $\operatorname{ker}(T)$, of $T$ again depends only on the image of $T$ in $\tilde{A}$, hence we can speak of the kernel, $\operatorname{ker}(l)$, of $l \in A$. We recall that if $A=C^{*}(G), \tilde{G}$ stands for $\tilde{A}$ (cf. $[3,18.5$, p. 323$\left.]\right)$. In this case we write $\tilde{G}_{\text {norm }}$ for $\tilde{A}_{\text {norm }}$.

THEOREM 1. Let $G$ be a connected Lie group. Then the map $l \mapsto \operatorname{ker}(l)$ from $\tilde{G}_{\text {norm }}$ into $\operatorname{Prim}(G)$ is a bijection.

Theorem 1 easily implies the following

COROLlaRY. If $g$ is an element, different from the unity, of $G$, there is a normal factor representation $T$ of $G$ such that $T(g)$ is not the unit operator.

Let $J$ be some element of $\operatorname{Prim}(G)$. Then by the above theorem there is a normal factor representation $T$ such that $\operatorname{ker}(T)=J$, and the character of $T$, denoted by $\chi_{J}$, up to a positive multiplicative constant depends only on $J$. Our next result, where we assume that $G$ is simply connected, gives an expression of $\chi_{J}$ in terms of type I traces. For its formulation we quote the following facts, which are either known or established during the proof of Theorem 1. Put $L=[G, G]$. $L$ is a closed, connected, invariant and type I subgroup of $G$ (cf. [4, 2.3, Proposition, p. 427]). Given $\pi \in \hat{L}$, there is a canonical choice of a closed subgroup $K_{\pi}$, containing $L$, of $G$, such that $\pi$ extends trivially to a representation $\rho$ of $K$, and $\operatorname{ind}_{K_{\pi} \uparrow G} \rho$ is a factor representation of $G$ (cf. $\left[9\right.$, p. 484]). Denote by $\mathfrak{G}(\pi) \subset \mathbb{K}_{\pi}$ the set of all extensions of $\pi$ to $K_{\pi}$ and put $\mathfrak{G}=\bigcup_{\pi \in \hat{L}} \mathfrak{G}(\pi)$. If $\rho$ is some element of $\mathfrak{5}$, we write $J(\rho) \in \operatorname{Prim}(G)$ for the kernel of the representation it induces in $G$. Then the map $\rho \mapsto J(\rho)$ from $\mathbb{G}$ into $\operatorname{Prim}(G)$ is a surjection. Set $\mathfrak{A}=\{\rho ; J(\rho)=J\}$. One can show that $K_{\pi}$, if $\pi=\rho \mid L$ and $\rho$ describes $\mathfrak{A}$, does not change; we denote it by $K . \mathfrak{A}$, as a subspace of $\mathbb{R}$, is locally 
compact Hausdorff, invariant under the canonical action of $G$ on $\widehat{K}$ and carries an essentially uniquely determined $G$-invariant positive Radon measure $\mu$. Write $\mathfrak{L}=C^{*}(L)$ and $R$ for the right regular representation of $L$ on $\mathfrak{L}$. Denote by $B$ the family of all those continuous maps from $G$ into $B$, for which (1) $f(l x)=R(l) f(x)(l \in L, x \in G)$, (2) $\|f(x)\|$, as a function on $G / L$, is of compact support. Let $d v$ be a Haar measure on $G / L$. Given $f \in B$ and a continuous unitary representation $U$ of $G$, the map $x \mapsto U(f(x)) U(x)$ is constant on cosets according to $L$, and there is a uniquely determined element $f^{\prime}$ of $A=C^{*}(G)$ such that $U\left(f^{\prime}\right)=$ $\int_{G / L} U(f(x)) U(x) d v$ for any such $U$. The map $f \mapsto f^{\prime}(f \in B)$ is a bijection of $B$ with a dense submanifold of $A$, and for any normal factor representation $T$, the set of operators of the form $T(f)\left(f \in B \cap A^{+}\right)$and of finite trace generates the same von Neumann algebra as does $T$. We observe finally, that $f \mid K$ $\left(f \in B \cap A^{+}\right)$identifies to a positive element of $C^{*}(K)$. Thus we have

THEOREM 2. Assuming that $\mu$ is properly normalized we have

$$
\chi_{J}(f)=\int_{\mathfrak{A}} \operatorname{Tr}(\rho(f \mid K)) d \mu(\rho) \quad\left(\leqq+\infty ; f \in B \cap A^{+}\right)
$$

where $\operatorname{Tr}($ ) stands for ordinary (type I) trace with the standard normalization.

3. Suppose next that $G$ is a connected and simply connected solvable Lie group with the Lie algebra $g$. Here there is a canonical identification between the quotient space $\mathfrak{5} / J$ and "generalized orbits" we introduced before (cf. [9, Proposition 7.1, p. 539], [11, III.6 and Proposition 1]), the collection of which we denote by $\mathfrak{S}$. The elements of $\mathfrak{S}$ are certain geometrical objects constructed by aid of $\mathfrak{g}^{\prime}$ (=dual of the underlying space of $g$ ) and the contragredient representation of $G$ on $g^{\prime}$. For instance, if $\mathfrak{g}$ is nilpotent we have simply $\mathfrak{S}=\mathfrak{g}^{\prime} / G$. There is a canonical procedure associating with each element 0 of $\mathfrak{S}$ a unitary equivalence class $F(0)$ of factor representations of $G$ (cf. [9, pp. 548-551]). The next theorem gives the precise characterization of their image in $\tilde{G}$.

THEOREM 3. With the previous notations, let $l(0)$ be the image of $F(0)$ in $\tilde{G}$. Then $l$ establishes a bijection between $\mathcal{S}$ and $\widetilde{G}_{\text {norm }}$.

For 0 in $\subseteq$ we put $J(0)=\operatorname{ker}(F(0))$; then the diagram

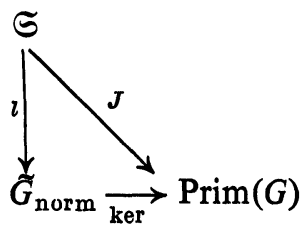


is commutative and thus $J: S \rightarrow \operatorname{Prim}(G)$ is a bijection. This was proved previously in [11, Theorem 1]).

\section{BIBLIOGRAPHY}

1. L. Auslander and B. Kostant, Polarization and unitary representations of solvable Lie groups, Invent. Math. 14 (1971), 255-354. MR 45 \#2092.

2. J. Dixmier, Sur les $C^{*}$-algebres, Bull. Soc. Math. France 88 (1960), 95-112. MR 22 \#12408.

3. - Les $C^{*}$-algèbres et leurs représentations, Cahiers Scientifiques, fasc. 29, Gauthier-Villars, Paris, 1964. MR 30 \#1404.

4. - Sur la représentation régulière d'un groupe localement compact connexe, Ann. Sci. Ecole Norm. Sup. (4) 2 (1969), 423-436. MR 41 \#5553.

5. J. Glimm, Type I $C^{*}$-algebras, Ann. of Math. (2) 73 (1961), 572-612. MR 23 \#A2066.

6. R. Godement, Sur la théorie des représentations unitaires, Ann. of Math. (2) 53 (1951), 68-124. MR 12, 421.

7. — Théorie des caractères. II. Définition et propriétés générales des caractères, Ann. of Math. (2) 59 (1954), 63-85. MR 15, 441.

8. A. Guichardet, Caracteres des algebres de Banach involutives, Ann. Inst. Fourier (Grenoble) 13 (1963), fasc. 1, 1-81. MR 26 \#5437; 30 \#1203.

9. L. Pukanszky, Unitary representations of solvable Lie groups, Ann. Sci. Ecole Norm. Sup. 4 (1971), 457-608.

10. - Action of algebraic groups of automorphisms on the dual of a class of type I groups, Ann. Sci. Ecole Norm. Sup. 5 (1972), 379-395.

11. - The primitive ideal space of solvable Lie groups, Invent. Math. 22 (1973), 75-118.

Department of Mathematics, University of Pennsylvania, Philadelphia, PenNSYlvania 19104 